\title{
Strand separation in negatively supercoiled DNA
}

\author{
Christian Mazza \\ LaPCS, UFR de Mathématiques \\ Université Claude Bernard Lyon-1 \\ Bâtiment Recherche B, Gerland \\ 50 Avenue Tony Garnier \\ 69366 Lyon Cedex 07, France. \\ e-mail: christian.mazza@univ-lyon1.fr
}

June 14, 2018

\begin{abstract}
We consider Benham's model for strand separation in negatively supercoiled circular DNA, and study denaturation as function of the superhelical density $\kappa<0$. We propose a statistical version of this model, based on bayesian segmentation methods of current use in bioinformatics; this leads to new algorithms with priors adapted to supercoiled DNA, taking into account the random nature of the free energies needed to denature hydrogen bonds.
\end{abstract}

Keywords: Strand separation, statistical mechanics, supercoiled DNA, Bayesian model

Running title: Strand separation 


\section{Introduction}

Initiation of transcription in DNA requires the two strands of the double helix to separate, and strand separation is enhanced in negative supercoiled DNA. Benham $(1979,1990,1996)$ proposes a mathematical model for the process of strand separation, based on statistical mechanics ideas, and develop algorithms to locate interesting sites along the DNA where strand separation or replication is strongly favored (see e.g. Clote and Backhofen(2000)). These computational methods are Metropolis dynamics (Sun et altri(1995)) or exact methods relying on transfer matrices(Fye and Benham(1999)). In a typical state, some hydrogen bonds are broken; we are interested in the repartition of the droplets of denatured bonds, and on the nature of the bases situated in these domains. Our aim is to investigate the effect of the number of droplets, of the degreee of negative superhelicity of the DNA and of the concentration in A+T bonds on the equilibrium properties of Benham's model. This is the topic of Section 2, where homopolymers are treated according to the number of connected domains of denatured bonds: in Section 2.1.1. we study denaturation when no restriction on the number of domains is imposed, and show that no robust and stable denatured state exists; this is the situation adapted to the algorithms of Fye and Benham(1999). In Section 2.1.2, we study the model when the number of domains $r_{N}$ is such that $r_{N} / N \rightarrow 0$, as $N \rightarrow \infty$, where $N$ denotes the number of bases of the DNA. We show the existence of a stable and robust denatured state when the duplex is sufficiently negatively supercoiled. This is the regime where the MCMC of Sun et altri applies. We next turn to heteropolymers in Section 2.2. and study localized denaturation as function of the proportion of $\mathrm{A}+\mathrm{T}$ bonds and of the level of negative superhelicity of the DNA. Section 3 focus on the statistical aspects of the model: Section 3.1 translates Benham's model in a Bayesian framework, and Section 3.2 shows how Bayesian segmentation methods of current use in bioinformatics, as presented in Liu and Lawrence(1999), can be of interest in the strand separation problem. This also give new algorithms with priors adapted to supercoiled DNA, which take into account the random fluctuations of the free energies needed to denature $\mathrm{A}+\mathrm{T}$ and $\mathrm{G}+\mathrm{C}$ bonds.

In what follows, we consider a spin system based on a circular graph with node set $S,|S|=N, N \in \mathbf{N}$, and, for each site $i \in S$, a spin $\sigma_{i} \in$ $\{-1,+1\}$. Benham's original model deals with a lattice gas, with binary random variables $n_{i}$. We use both notations by setting $n_{i}=\left(\sigma_{i}+1\right) / 2$, and use spins to link this model with known mean field models. The meaning of $n_{i}=0$ (resp. $\sigma_{i}=-1$ ) is that the bases of the double helix at site $i \in S$ are linked by an hydrogen bond, the link is closed, and $n_{i}=1$ (resp. $\sigma_{i}=+1$ ) means that this bond is broken, the link is open. Then $n:=\sum_{i=1}^{N} n_{i}$ denotes the number of open bonds. The partitioning of the 
DNA in domains allows the linking numbers to be regulated, where the linking number $L$ of a configuration describes the way the duplex winds about the axis, assuming that the axis of the helix is planar. The twist $T$ is the number of times the duplex revolves about its axis(see e.g. Clote and Backhofen(2000), chap. 6.2). When the DNA is relaxed, the so-called B-DNA state, a segment of $N$ bases produces typically the characteristic linking number $L_{0}=N / A$, where the constant $A$ is experimentally situated around 10.4. A negative supercoilded DNA is a configuration obtained from the B-DNA by cutting the strands using topoisomerases of type II; the strands then rotate around each others in the direction opposite of the twist of the helix, reducing then its linking number $\left(L<L_{0}\right)$. This forces then the circular axis of the helix to wind, producing then a more twisted and compact configuration(see e.g. Lewin(1994)). This supercoiled state permits for example to put the helix in nuclei. Benham's model permits to quantify the way supercoiling enhances strand separation.

Consider a supercoiled DNA with negative linking difference $\alpha=L-$ $L_{0}<0$, imposed during the process. Assume that $n$ links between bases are broken; the helix unwinds locally and thus increases its linking number to $\alpha+n / A$. Because the strands car rotate around each others, the same process induced also a twist $\mathcal{T}$, yielding a residual linking difference $\alpha_{r}=$ $\alpha+n / A-\mathcal{T}$. The total twist $\mathcal{T}$ between separated regions is modeled as

$$
\mathcal{T}=\sum_{i=1}^{N} \frac{n_{i} \tau_{j}}{2 \pi}
$$

where $\tau_{i} \in \mathbf{R}$ is the local helicity. Benham's idea is to quantify all of these steps with free energy costs. The torsional free energy $G_{t}$ is given by

$$
G_{t}=\frac{C}{2} \sum_{i=1}^{N} n_{i} \tau_{i}^{2}
$$

for some stiffness coefficient $C>0$. Consider a configuration in which $n$ bonds are separated in $r$ runs, that is in $r$ connected components of open bonds. In what follows, $2 r$ is the perimeter of the configuration.

The free energy cost for separation is modeled as

$$
G_{s}=a r+\sum_{i=1}^{N} b_{i} n_{i}, a>0, r=\sum_{i} n_{i}\left(1-n_{i+1}\right),
$$

where the parameters $b_{i}$ indicate the natures of the bases located at sites $i \in S: A T$ links are formed of 2 hydrogen bonds, and GC links consist in 3 hydrogen bonds. If $i \in S$ is associated with an AT link, we set $b_{i}=b_{A T}$ and $b_{i}=b_{G C}$ otherwise, with $b_{G C}>b_{A T}$. In the homopolymer case, $b_{i} \equiv b>0$. This will act as an exterior magnetic field in the statistical approach; when 
the bases are chosen at random on the DNA with some law, $\sum_{i} n_{i} b_{i}$ can be viewed as a random external field. The free energy $b$ needed to break the hydrogen bonds, and thus to separate a base pair, depends on the inverse temperature $\beta$ :

$$
b=b(\beta)=\triangle H\left(1-\frac{\beta_{m}}{\beta}\right),
$$

where $\triangle H$ is the enthalpy of the reaction and $\beta_{m}$ is the inverse temperature associated to the melting temperature $T_{m}$. Below $T_{m}$, the field $b$ is positive, this is the regime we are interested in. From Benham(1992), the pBR322 DNA is such that $\triangle H_{A T}=7.25 \mathrm{kcal} / \mathrm{mol}, \triangle H_{G C}=9.02 \mathrm{kcal} / \mathrm{mol}$, and the melting temperature $T_{m}$ follows the law

$$
T_{m}=354.55+16.6 \log (x)+41 F_{G C},
$$

where $x$ is some parameter and $F_{G C}=0$ for AT bonds and $F_{G C}=1$ for GC bonds. When $x=0.01$ and $T=\beta^{-1}=310 \mathrm{~K}$, the resulting free energies are given by $b_{A T}=0.255 \mathrm{kcal} / \mathrm{mol}$ and $b_{G C}=1.301 \mathrm{kcal} / \mathrm{mol}$.

Long range interactions appear with the fluctuations of the linking number: it is known experimentally that the energy cost associated with the residual linking number for supercoiled DNA is given by

$$
G_{r}=\frac{K \alpha_{r}^{2}}{2}=\frac{K}{2}\left(\alpha+\frac{n}{A}-\mathcal{T}\right)^{2} .
$$

Experimentally, the coefficient $K$ is inverse proportional to the number of bases of the DNA; we thus set

$$
K=\frac{K_{0}}{N}
$$

As $N$ is large, basic statistical reasoning suggests to renormalize the variable $n$ as $n / N$, to catch the thermodynamical limit. We thus introduce the superhelical density $\kappa$ by setting

$$
\alpha=\kappa N \text {. }
$$

The overall free energy takes then the form

$G=\frac{C}{2} \sum_{i=1}^{N} n_{i} \tau_{i}^{2}+N \frac{K_{0}}{2}\left(\kappa+\frac{n}{2 N A}-\frac{1}{N} \sum_{i=1}^{N} \frac{n_{i} \tau_{i}}{2 \pi}\right)^{2}+\sum_{i=1}^{N}\left(\left(a+2 b_{i}\right) n_{i}-a n_{i} n_{i+1}\right)$.

We use the local fields $2 b_{i}$ insteed of $b_{i}$ for notational purpose. 


\section{Results on denaturation}

\subsection{The homopolymer approximation}

In this paragraph, we suppose that $b_{i} \equiv b>0$ and that $\tau_{i} \equiv \tau \in \mathbf{R}$. Set for convenience

$$
M_{N}=\frac{1}{N} \sum_{i=1}^{N} n_{i}, m_{N}=\frac{1}{N} \sum_{i=1}^{N} \sigma_{i}=2 M_{N}-1 .
$$

Then the Hamiltonian of the system becomes

$$
G_{\tau}=N\left(2 b M_{N}+\frac{C \tau^{2}}{2} M_{N}+\frac{K_{0}}{2}\left(\kappa+\left(\frac{1}{2 A}-\frac{\tau}{2 \pi}\right) M_{N}\right)^{2}\right)+a H_{\text {sing }} / 4,
$$

where the index $\tau$ of $G_{\tau}$ indicates the dependence on the torsion coefficient $\tau$ and $H_{\text {sing }}$ denotes the Hamiltonian associated with the nearest neighbor ferromagnetic Ising model in dimension 1

$$
H_{\text {sing }}=-\sum_{i=1}^{N-1} \sigma_{i} \sigma_{i+1}=4 r-N .
$$

Before introducing the Gibbs measure of the system at inverse temperature $\beta>0$, let us proceed as in Benham by averaging the system with respect to the torsion coefficient $\tau$. The Boltzman weight should be $\exp \left(-\beta G_{\tau}\right)$. Averaging over $\tau \in \mathbf{R}$ gives the integral $\int_{\mathbf{R}} \exp \left(-\beta G_{\tau}\right) d \tau$, that is the effective Hamiltonian

$$
H_{1}=N 2 b M_{N}+\frac{a}{4} H_{\text {sing }}+N \frac{2 \pi^{2} C K_{0}}{4 \pi^{2} C+K_{0} M_{N}}\left(\kappa+\frac{M_{N}}{A}\right)^{2},
$$

(see Fye and Benham(1999)).

\subsubsection{Arbitrary large perimeter}

In this approximation, we shall consider the behavior of $M_{N}$ in the thermodynamical limit $N \rightarrow \infty$ under Gibbs measure

$$
\pi_{\beta, B}(\sigma)=\exp \left(-\beta H_{1}(\sigma)\right) / Z_{N}(\beta, B)
$$

where $Z_{N}(\beta, B)$ denotes the related partition function, and where $B=$ $\left(b_{i}\right)_{1 \leqslant i \leqslant N}, b_{i} \equiv b$, denotes the exterior field. We use mainly Laplace method bu using the large deviation rate function associated with the ferromagnetic nearest neighbor Ising model in dimension one. Notice the appearance of the magnetization $M$ in the denominator of (2), which is quite unconventional. 
Let $\pi_{\beta_{a}}$ be the Gibbs measure at inverse temperature $\beta_{a}=a \beta / 4$ associated

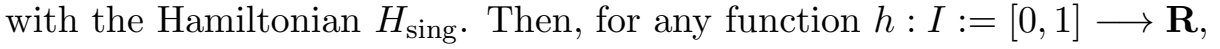

$$
<h\left(M_{N}\right)>_{\pi_{\beta, B}}=\frac{<h\left(M_{N}\right) \exp \left(-N \beta F_{B}\left(M_{N}\right)\right)>_{\pi_{\beta a}}}{<\exp \left(-N \beta F_{B}\left(M_{N}\right)\right)>_{\pi_{\beta_{a}}}},
$$

where

$$
F_{B}(y)=b(2 y-1)+G(y), y \in \mathrm{I},
$$

and

$$
G(y)=\frac{2 \pi^{2} C K_{0}}{4 \pi^{2} C+K_{0} y}\left(\kappa+\frac{y}{A}\right)^{2} .
$$

Let $\mu_{N}$ be the law of $m_{N}(\sigma)=2 M_{N}(\sigma)-1$ under Gibbs measure $\pi_{\beta_{a}}$. Then

$$
<h\left(M_{N}\right)>_{\pi_{\beta, B}}=\frac{\int_{-1}^{+1} \mu_{N}(\mathrm{dz}) h\left(\frac{1+z}{2}\right) \exp \left(-\beta N F_{B}\left(\frac{1+z}{2}\right)\right)}{\int_{-1}^{+1} \mu_{N}(\mathrm{dz}) \exp \left(-\beta N F_{B}\left(\frac{1+z}{2}\right)\right)} .
$$

Benham(1979) investigates the thermodynamics of supercoiled DNA, by minimizing free energies, and introduces critical thresholds of supercoiling. In this macroscopic approach, it is shown that sufficient negative supercoiling implies local denaturation. In Benham(1996), this work is extended to positively supercoiled DNA $(\kappa>0)$; looking at the various plots contained in this work, we see the appearance of critical superhelical densities above which a positively supercoiled DNA remains intact at temperatures higher than the melting point. Similarly, we introduce the

Definition 1 The order parameter of the system is

$$
<M_{N}>_{\pi_{\beta, B}}, \text { or }<m_{N}>_{\pi_{\beta, B}},
$$

the magnetization of the spin system. We say that the system exhibits phase transitions when there exist critical superhelical densities $\bar{\kappa}_{c}(B)>$ $\kappa_{c}(B)$ such that, in the large $N$ limit, $\left\langle M_{N}>_{\pi_{\beta, B}} \rightarrow 0\right.$ as $\kappa>\bar{\kappa}_{c}(B)$, $<M_{N}>_{\pi_{\beta, B}} \rightarrow 1$ as $\kappa<\kappa_{c}(B)$, and lim $<M_{N}>_{\pi_{\beta, B}} \in(0,1)$ otherwise.

Let $\Lambda_{N}(\lambda)$ be the logarithmic moment generating function

$$
\Lambda_{N}(\lambda):=\ln \left(\pi_{\beta_{a}}\left(\exp \left(N m_{N} \lambda\right)\right)\right),
$$

with (see e.g. Baxter(1982), p.34)

$$
\Lambda_{\infty}(\lambda):=\lim _{N \rightarrow \infty} \frac{1}{N} \Lambda_{N}(\lambda)=\ln \left(\frac{e^{\beta_{a}} \cosh (\lambda)+\sqrt{e^{2 \beta_{a}} \sinh (\lambda)^{2}+e^{-2 \beta_{a}}}}{e^{\beta_{a}}+e^{-\beta_{a}}}\right) .
$$

Then, according to Gaertner-Ellis Theorem, (see e.g. Dembo and Zeitouni(1992)) the law of $m_{N}$ under $\pi_{\beta_{a}}$ satisfies a large deviation principle with good rate function

$$
I_{\text {sing }}(z)=\sup _{\lambda \in \mathbf{R}}\left(\lambda z-\Lambda_{\infty}(\lambda)\right)
$$


the Legendre transform of $\Lambda_{\infty} . \quad \Lambda_{\infty}^{\prime}=e^{\beta_{a}} \sinh / \sqrt{e^{2 \beta_{a}} \sinh ^{2}+e^{-2 \beta_{a}}}$, and $\Lambda_{\infty}^{\prime \prime}=e^{\beta_{a}} \cosh /\left(e^{2 \beta_{a}} \sinh ^{2}+e^{-2 \beta_{a}}\right) . \Lambda_{\infty}$ is thus strictly convex on $\mathbf{R}$, and its Legendre transform is essentially smooth (see Theorem 26.3 in Rockafellar(1972)). The derivative $I_{\text {sing }}^{\prime}(z)$ tends to $+\infty$ when $z$ converges to a boundary point of the domain of $I_{\text {sing }}$. From computation,

$$
\begin{aligned}
I_{\operatorname{sing}}(z)= & z \ln \left(\frac{z e^{-2 \beta}+\sqrt{1+z^{2}\left(e^{-4 \beta_{a}}-1\right)}}{\sqrt{1-z^{2}}}\right) \\
& -\ln \left(\frac{e^{\beta_{a}} \sqrt{1+z^{2}\left(e^{-4 \beta_{a}}-1\right)}+e^{-\beta_{a}}}{\sqrt{1-z^{2}}\left(e^{\beta_{a}}+e^{-\beta_{a}}\right)}\right),
\end{aligned}
$$

$|z| \leqslant 1$, and $I_{\text {sing }}(z)=+\infty$ when $|z|>1$.

In the special case where $a=0$, the rate function becomes the entropy

$$
I_{\text {sing }}(z)=\frac{1+z}{2} \ln (1+z)+\frac{1-z}{2} \ln (1-z),|z| \leqslant 1 .
$$

The integrals appearing in (6) can be estimated through Laplace's method by rewritting the numerator heuristically as

$$
\int_{-1}^{+1} \mathrm{dzh}\left(\frac{1+z}{2}\right) \exp (-N J(z))
$$

where we set

$$
J(z):=I_{\text {sing }}(z)+\beta F_{B}\left(\frac{1+z}{2}\right),|z| \leqslant 1 .
$$

Then (6) is asymptotically equivalent to

$$
\frac{\int_{-1}^{+1} \mathrm{dzh}\left(\frac{1+z}{2}\right) \exp \left(-N\left(J(z)-\inf _{|z| \leqslant 1} J(z)\right)\right)}{\int_{-1}^{+1} \mathrm{dz} \exp \left(-N\left(J(z)-\inf _{|z| \leqslant 1} J(z)\right)\right)} .
$$

Theorem $1 \exists$ a unique $z_{*} \in(-1,+1)$ minimizing $J(z)$, with

$$
<M_{N}>_{\pi_{\beta, B}} \longrightarrow \frac{1+z_{*}}{2} .
$$

The model does not exhibit phase transitions in the sense of Definition 11. This is a consequence of the stiffness of the rate function $I_{\operatorname{sing}}(z)$ as $|z| \rightarrow 1: \lim _{|z| \rightarrow 1}(\mathrm{~d} / \mathrm{dz}) I_{\text {sing }}(z)=+\infty$, and the rate function $J(z)$ can not be decreasing in the neighborhood of $z=1$. The minima of $J(z)$ are thus located in the interior of the unit interval. Suppose that the Ising measure is replaced by some probability measure $V_{N}$ on $\Omega_{N}$, such that the law of $M_{N}$ under $V_{N}$ satisfies a large deviation principle with strictly convex and smooth free energy function $\Lambda$; then its Legendre transform is essentially smooth, and again, using Varadhan's Theorem, one gets the rate function 
$J(z)-\inf _{z} J(z)$; similarly, the minima of $J$ are located in the interior of the domain of the Legendre transform, and no phase transition occurs.

Proof: Consider the probability measure

$$
\nu_{N}(C)=\frac{\int_{C} \mu_{N}(\mathrm{~d} z) \exp \left(-N \beta F_{B}((1+z) / 2)\right.}{\int_{-1}^{1} \mu_{N}(\mathrm{~d} z) \exp \left(-N \beta F_{B}((1+z) / 2)\right.},
$$

for any Borel subset $C$ of $[-1,1]$. Varadhan's Theorem (see Deuschel and Stroock, Theorem 2.1.10 and exercice 2.1.24) gives that $\nu_{N}$ satisfies a large deviation principle with good rate function $J(z)$. If $J$ attains its infimum at a unique point $z_{*}$ of the interval, the sequence $\nu_{N}$ converges weakly to the point mass $\delta_{z_{*}}$. Consider first $F_{B}(y), y \in[0,1]$, or equivalently $F_{B}+2 b \kappa A+b$, which is equal to

$$
\frac{2\left(b A^{2}+\pi^{2} C\right)}{A^{2}} \frac{\left(y-y_{0}\right)\left(y-y_{1}\right)}{\left(y-y_{2}\right)},
$$

where

$$
y_{0}=-\kappa A>0, y_{1}=-\frac{\pi^{2} C A}{K_{0}\left(b A^{2}+\pi^{2} C\right)}\left(4 b A+K_{0} \kappa\right) \text { and } y_{2}=-\frac{4 \pi^{2} C}{K_{0}} .
$$

Notice that $b>0$ implies that $y_{1}>y_{2}$. The function has a pole at $y=y_{2}<$ 0 , and two roots $y_{1}$ and $y_{0}>0$. Then $F_{B}$ is strictly convex on the half line $\left(y_{2},+\infty\right)$. Thus $J$ is strictly convex on $[-1,1]$, with $\lim _{|z| \rightarrow 1} J^{\prime}(z)=+\infty$. The unique infimum is thus located in the interior of the interval.

\subsubsection{Limited perimeter}

Computations done in some theoretical studies (see e.g. Benham(1989), p. 268 and Benham(1990), p. 6302) or empirical studies( see e.g. Sun et altri(1995, p. 8658)) deal with the behavior of $M_{N}$ when $2 r$ is fixed, or is small. In what follows, we give conditions on the growth of $r=r_{N}$ ensuring the possibility of phase transitions, that is the possibility for the existence of a stable and robust denatured state when the superhelical density is small enough.

In what follows, we condition on the event $\left\{\sigma ;|\sigma|=2 r_{N}\right\}$, where for any configuration $\sigma,|\sigma|$ denotes the perimeter of $\sigma$, with $|\sigma|=\left|i ; \sigma_{i}=-\sigma_{i+1}\right|=$ $2 r$, and $H_{\text {sing }}(\sigma)=2|\sigma|-N$. Classical combinatorics (see e.g. Feller(1971)) shows that the number of configurations of length $N$ with $\sum_{i=1}^{N} n_{i}=n$ and perimeter $1 \leqslant r \leqslant N / 2$ is given by

$$
M(n, r)=\frac{N}{r}\left(\begin{array}{c}
n-1 \\
r-1
\end{array}\right)\left(\begin{array}{c}
N-n-1 \\
r-1
\end{array}\right) .
$$


Let $U_{r, N}$ be the uniform probability measure on the subset $\mathcal{C}_{r, N}$ of the cube $\Omega_{N}$ consisting of spins of perimeter $2 r$. Let $P_{r, N}$ be the law of $M_{N}$ under $U_{r, N}$, with $\operatorname{supp}\left(P_{r, N}\right)=\{r / N, \cdots, 1-r / N\}$, given by

$$
P_{r, N}(n / N)=\frac{\left(\begin{array}{c}
n-1 \\
r-1
\end{array}\right)\left(\begin{array}{c}
N-n-1 \\
r-1
\end{array}\right)}{\sum_{r \leqslant n \leqslant N-r}\left(\begin{array}{c}
n-1 \\
r-1
\end{array}\right)\left(\begin{array}{c}
N-n-1 \\
r-1
\end{array}\right)} .
$$

Then the average $\left\langle h(M)>_{\pi_{\beta, B}}\right.$ becomes

$\frac{\sum_{r} \exp \left(-\beta_{a} r\right)|\{|\sigma|=2 r\}| \sum_{r \leqslant n \leqslant N-r} P_{r, N}(n / N) h(n / N) \exp \left(-N \beta F_{B}(n / N)\right)}{\sum_{r} \exp \left(-\beta_{a} r\right)|\{|\sigma|=2 r\}| \sum_{r \leqslant n \leqslant N-r} P_{r, N}(n / N) \exp \left(-N \beta F_{B}(n / N)\right)}$

We will be concerned with integrals of the form

$$
\int_{0}^{1} P_{r_{N}, N}(\mathrm{~d} y) h(y) \exp \left(-\beta N F_{B}(y)\right)
$$

when $r=r_{N}$ is such that $r_{N} / N \longrightarrow 0$ as $N \rightarrow \infty$.

Lemma 1 Assume that $2 r_{N}<N$ and that $r_{N} / N \longrightarrow 0$ as $N \rightarrow \infty$. Then

$$
\frac{1}{N} \log \left(\begin{array}{c}
n \\
r_{N}
\end{array}\right) \longrightarrow 0, N \rightarrow \infty
$$

when $n=[\rho N]$, for $0<\rho \leqslant 1$. The sequence of probability measures $\left(P_{r_{N}, N}\right)_{n \in \mathbf{N}}$ satisfies a large deviation principle with good rate function $I^{r}$ : $\mathbf{R} \longrightarrow[0,+\infty)$ given by $I^{r}(y)=0, y \in I$, and $I^{r}(y)=+\infty, y \in I^{c}$.

Proof: The first assertion is a consequence of Stirling's formula. Assume that $A=(a, b) \subset I$. For $N$ large enough, $A \cap\left\{r_{N} / N, \cdots, 1-r_{N} / N\right\} \neq \emptyset$, and $A$ contains an element $\rho_{N}$ of the form $\rho_{N}=[\rho N] / N$ for some $0<\rho<1$, with

$$
\log P_{r_{N}, N}\left(\left\{\rho_{N}\right\}\right) \leqslant \log P_{r_{N}, N}(A) \leqslant 0 .
$$

Using (77) and (8), it remains to check that

$$
\frac{1}{N} \log \left(\sum_{r \leqslant n \leqslant N-r}\left(\begin{array}{l}
n \\
r
\end{array}\right)\left(\begin{array}{c}
N-n \\
r
\end{array}\right)\right) \longrightarrow 0 .
$$

But, as the sum larger than one,

$$
\begin{gathered}
0 \leqslant \frac{1}{N} \log \left(\sum_{r \leqslant n \leqslant N-r}\left(\begin{array}{c}
n \\
r
\end{array}\right)\left(\begin{array}{c}
N-n \\
r
\end{array}\right)\right) \\
\leqslant \frac{1}{N} \log \left((N-2 r) \sup _{r \leqslant n \leqslant N-r}\left(\begin{array}{c}
n \\
r
\end{array}\right)\left(\begin{array}{c}
N-n \\
r
\end{array}\right)\right)
\end{gathered}
$$




$$
\leqslant \frac{\log (N)}{N}+\frac{1}{N} \log \left(\sup _{r \leqslant n \leqslant N-r}\left(\begin{array}{l}
n \\
r
\end{array}\right)\left(\begin{array}{c}
N-n \\
r
\end{array}\right)\right) .
$$

The sequence $\left(\left(\begin{array}{c}n \\ r\end{array}\right)\left(\begin{array}{c}N-n \\ r\end{array}\right)\right)_{n}$ attains its supremum when $n=[N / 2]$, and the statement is a consequence of (8). $A$ is a $I^{r}$-continuity set. When $A \cap I=\emptyset$, $P_{r_{N}, N}(A) \equiv 0$, which is consistent with $I^{r}(y)=+\infty$ when $y \notin I$. When $A$ takes the form $A=[-c, \varepsilon]$ with $c>0$ and $\varepsilon>0, \exists N_{0} \in \mathbf{N}$ such that $0<r_{N} / N<\varepsilon, \forall N \geqslant N_{0}$, and the same argument applies. When $A=[-c, 0], P_{r_{N}, N}(A) \equiv 0$, and we have the inequalities defining the large deviation principle

$$
-\inf _{y \in A^{0}} I^{r}(y) \leqslant \liminf \frac{1}{N} \log \left(P_{r_{N}, N}(A)\right)
$$

and

$$
\limsup \frac{1}{N} \log \left(P_{r_{N}, N}(A)\right) \leqslant-\inf _{y \in \bar{A}} I^{r}(y) .
$$

The above arguments show that the upper and lower bounds (9) hold for open and compact sets. The sequence of measures $\left(P_{r_{N}, N}\right)$ is supported by the unit interval, and the sequence is exponentially tight. The large deviation principle follows.

When the large deviation principle is satisfied with the flat rate function $I^{r}$, Laplace's method gives that the system exhibits a phase transition with respect to the order parameter $M_{N}$ : the mass of the integral is located near the infimum of the function $F_{B}(y)$, and therefore, the DNA is completely denatured when the parameters of the problem are such that

$$
\inf _{y \in(0,1)} F_{B}(y)=F_{B}(1)
$$

This occurs for example when the helicity density $\kappa$ is smaller than a critical density $\kappa_{c}$.

Theorem 2 The function $F_{B}: I \longrightarrow \mathbf{R}$ attains its infimum at a unique point $y_{*}(B, \kappa)$. Let

$$
y_{0}=-\kappa A>0, y_{1}=-\frac{\pi^{2} C A}{K_{0}\left(b A^{2}+\pi^{2} C\right)}\left(4 b A+K_{0} \kappa\right) \text { and } y_{2}=-\frac{4 \pi^{2} C}{K_{0}} .
$$

Let $\bar{\kappa}_{c}(B)$ and $\kappa_{c}(B)$ be the smallest roots of the polynomials $P(\kappa)=\left(y_{0}-\right.$ $\left.y_{2}\right)\left(y_{1}-y_{2}\right)-y_{2}^{2}$ and $Q(\kappa)=P(\kappa)-\left(1-2 y_{2}\right)$, with $\bar{\kappa}_{c}(B)>\kappa_{c}(B)$. Set $M_{*}=y_{2}+\sqrt{\left(y_{2}-y_{0}\right)\left(y_{2}-y_{1}\right)}$. Then i) $\kappa>\bar{\kappa}_{c}(B)$ implies that $F_{B}$ is increasing on I with $y_{*}(B, \kappa)=0$, ii) $\kappa_{c}(B)<\kappa<\bar{\kappa}_{c}(B)$ implies that $y_{*}(B, \kappa)=M_{*} \in(0,1)$, with $F_{B}$ decreasing below $y_{*}(B, \kappa)$ and increasing above $y_{*}(B, \kappa)$, and iii) $\kappa<\kappa_{c}(B)$ implies that $F_{B}$ is decreasing on I with 
$y_{*}(B, \kappa)=1$. Let $h: \mathrm{I} \longrightarrow \mathbf{R}$ be bounded and continuous. Assume that $r_{N} / N \longrightarrow 0$ as $N \rightarrow \infty$. Then

$$
\frac{P_{r_{N}, N}\left(h \exp \left(-N \beta F_{B}\right)\right)}{P_{r_{N}, N}\left(\exp \left(-N \beta F_{B}\right)\right)} \longrightarrow h\left(y_{*}(B, \kappa)\right) .
$$

Proof: We look for the infimum of $F_{B}$ on I; equivalently, we can consider the infimum of $F_{B}+2 b \kappa A+b$, which is equal to

$$
\frac{\left(2 b A^{2}+2 \pi^{2} C\right)}{A^{2}} \frac{\left(y-y_{0}\right)\left(y-y_{1}\right)}{\left(y-y_{2}\right)} .
$$

Notice that $b>0$ implies that $y_{1}>y_{2}$. The function has a pole at $y=$ $y_{2}<0$, and two roots $y_{1}$ and $y_{0}>0$. We see that the first part of the theorem (cases i), ii) and iii)) is related to the location of the infimum of the function with respect to $\left(y_{2}, 0\right),(0,1)$ and $(1, \infty)$. Taking the derivative, we must look for the roots of $y^{2}-2 y_{2} y+y_{2}\left(y_{0}+y_{1}\right)-y_{0} y_{1}$, of discriminant $\left(y_{0}-y_{2}\right)\left(y_{1}-y_{2}\right) \geqslant 0$, since $y_{1}>y_{2}, y_{0}>0$ and $y_{2}<0$, and we get the condition for the largest root $M_{*}$. The polynomial $P(\kappa)$ is obtained by imposing $M_{*}<0$, and $Q(\kappa)$ by imposing $M_{*}<1$. Concerning the last assertion, consider the probability measure

$$
\mu_{N}(A):=\frac{\int_{A} \exp \left(-N \beta F_{B}(y)\right) P_{r_{N}, N}(\mathrm{~d} y)}{\int_{I} \exp \left(-N \beta F_{B}(y)\right) P_{r_{N}, N}(\mathrm{~d} y)},
$$

for any Borel subset $A$. From Varadhan's Theorem (see e.g. Theorem 2.1.10 and exercice 2.1.24 in Deuschel and Stroock(1989) or Theorem II.7.2 in Ellis(1985)) and Lemma 1 the sequence $\left(\mu_{N}\right)$ satisfies a large deviation principle with rate function $I_{F}(y)-\inf _{y} I_{F}(y)$, where $I_{F}(y)=I^{r}(y)+\beta F_{B}(y)$. $\inf _{y \in \mathbf{R}} I_{F}(y)=\beta \inf _{y \in \mathrm{I}} F_{B}(y)$, which is realized at a unique element $y_{*}(B, \kappa)$; $\mu_{N}$ converges then weakly to the point mass $\delta_{y_{*}(B, \kappa)}$.

\subsection{The heteropolymer Case}

In the heteropolymer case, the field $B^{N}=\left(b_{i}\right)_{1 \leqslant i \leqslant N}$ is indexed by a word of length $N$ on the two letters alphabet $\{A+T, G+C\}$. Given $B^{N}$, let

$$
\rho_{N}^{+}\left(B^{N}\right):=\frac{1}{N} \sum_{i=1}^{N} \mathrm{I}\left(b_{i}=b_{A T}\right)
$$

where $\mathrm{I}(\cdot)$ is the indicator function. Given some proportion $\rho^{+} \in \mathrm{I}$, we shall consider families $\left(B^{N}\right)_{N \in \mathbf{N}}$ of words with $\rho_{N}^{+}\left(B^{N}\right) \longrightarrow \rho^{+}$. Let

$$
m_{N}^{+}(\sigma)=\frac{1}{N} \sum_{i=1}^{N} \mathrm{I}\left(b_{i}=b_{A T}\right) \sigma_{i} \text { and } m_{N}^{-}(\sigma)=\frac{1}{N} \sum_{i=1}^{N} \mathrm{I}\left(b_{i}=b_{G C}\right) \sigma_{i}
$$


with $m_{N}=m_{N}^{+}+m_{N}^{-}$, and consider the mapping $\Psi_{B^{N}}: \Omega_{N} \longrightarrow \mathbf{R}^{2}$ given by $\Psi_{B^{N}}(\sigma)=\left(m_{N}^{+}(\sigma), m_{N}^{-}(\sigma)\right)$, which permits to control the eventual localization of the magnetization (see Mathieu and Picco(1998) for other use of this mapping in random fields Curie Weiss models). Let $V_{N}$ be a probability measure on $\Omega_{N}$, and let $Q_{N}$ be the image measure of $V_{N}$ under $\Psi_{B^{N}}$. We consider the behavior of $m_{N}^{+}$and $m_{N}^{-}$under the Gibbs measure

$$
\pi_{\beta, B^{N}, V_{N}}(\sigma)=\frac{V_{N}(\sigma) \exp \left(-N \beta F_{B^{N}}(\sigma)\right)}{Z_{N}\left(\beta, B^{N}, V_{N}\right)},
$$

where we set

$$
F_{B^{N}}(\sigma)=G\left(M_{N}(\sigma)\right)+\frac{1}{N} \sum_{i=1}^{N} b_{i} \sigma_{i} .
$$

Let us denote by $B_{G C}$ and $B_{A T}$ the fields associated with the GC and AT homopolymers, with critical superhelical densities $\bar{\kappa}_{c}\left(B_{G C}\right), \kappa_{c}\left(B_{G C}\right)$, $\kappa_{c}\left(B_{A T}\right)$ and limiting proportion of broken bonds $y_{*}\left(B_{G C}, \kappa\right)$ (see Theorem 2).

Theorem 3 Let $\left(B^{N}\right)_{N \in \mathbf{N}}$ be a sequence of words of $\left\{b_{A T}, b_{G C}\right\}^{N}$ with $\rho_{N}^{+}\left(B^{N}\right) \longrightarrow \rho^{+}$, for some $\rho^{+} \in$ I. Suppose that the family of probability measures $Q_{N}$ satisfies a large deviation principle with rate function $I_{D}$ : $\mathbf{R}^{2} \longrightarrow[0,+\infty)$ given by $I_{D}(y)=0, y \in D$ and $I_{D}(y)=+\infty, y \in D^{c}$, where $D=\left[-\rho^{+}, \rho^{+}\right] \times\left[-\rho^{-}, \rho^{-}\right]$. Let $\kappa$ be such that $\kappa_{c}\left(B_{G C}\right)<\kappa<\kappa_{c}\left(B_{A T}\right)$. Assume that $\rho^{+}>y_{*}\left(B_{G C}, \kappa\right)$ : Then denaturation is localized on the AT domain, that is $<m_{N}^{+}>_{\pi_{\beta, B^{N}, V_{N}}} \longrightarrow \rho^{+}$, and $<m_{N}^{-}>_{\pi_{\beta, B^{N}, V_{N}}} \longrightarrow-\rho^{-}$, where we set $\rho^{-}=1-\rho^{+}$, the proportion of GC bonds. Conversely, assume that $\rho^{+}<y_{*}\left(B_{G C}, \kappa\right)$ : Then $<m_{N}^{+}>_{\pi_{\beta, B^{N}, V_{N}}} \longrightarrow \rho^{+}$, and $<m_{N}^{-}>_{\pi_{\beta, B^{N}, V_{N}}} \longrightarrow$ $2 y_{*}\left(B_{G C}, \kappa\right)-1-\rho^{+}>-\rho^{-}$.

Remark 1 If the parameters of the problem are such that $\bar{\kappa}_{c}\left(B_{G C}\right)<\kappa_{c}\left(B_{A T}\right)$ (recall that the fields are temperature dependent, see (11)), and $\kappa$ is so that $\bar{\kappa}_{c}\left(B_{G C}\right)<\kappa<\kappa_{c}\left(B_{A T}\right)$, denaturation is localized on the AT domain, for arbitrary proportion $\rho^{+}$of $A T$ bonds. However, when $\kappa<\bar{\kappa}_{c}\left(B_{G C}\right)$, denaturation is localized on the AT domain when $\rho^{+}>y_{*}\left(B_{G C}, \kappa\right)$ and denaturation expands beyond the AT domain when $\rho^{+}<y_{*}\left(B_{G C}, \kappa\right)$.

Proof: We must evaluate the asymptotic behavior of

$$
\frac{\sum_{\sigma} V_{N}(\sigma) \exp \left(-N \beta F_{B^{N}}(\sigma)\right) h\left(m_{N}^{+}(\sigma), m_{N}^{-}(\sigma)\right)}{\sum_{\sigma} V_{N}(\sigma) \exp \left(-N \beta F_{B^{N}}(\sigma)\right)},
$$

for functions $h$ of the two variables $\left(m_{N}^{+}, m_{N}^{-}\right)$. Notice that

$$
\begin{aligned}
F_{B^{N}}(\sigma) & =G\left(M_{N}(\sigma)\right)+b_{A T} m_{N}^{+}(\sigma)+b_{G C} m_{N}^{-}(\sigma) \\
& =G\left(M_{N}(\sigma)\right)+b_{G C} m_{N}(\sigma)+\left(b_{A T}-b_{G C}\right) m_{N}^{+}(\sigma)
\end{aligned}
$$


Let $F\left(m^{+}, m^{-}\right)=G(M)+b_{A T} m^{+}+b_{G C} m^{-}$, where $m=m^{+}+m^{-}$and $M=(m+1) / 2$. We must thus check the behavior of the expectation

$$
\mu_{N}(h)=\frac{\int h(y) \exp (-N \beta F(y)) Q_{N}(\mathrm{dy})}{\int \exp (-N \beta F(y)) Q_{N}(\mathrm{dy})},
$$

for bounded and continuous functions $h$. From Varadhan's Theorem, the family of probability measures $\mu_{N}$ satisfies a large deviation principle with rate function $I_{F}(y)-\inf _{y} I_{F}(y)$, where $I_{F}(y)=I_{D}(y)+\beta F(y) \cdot \inf _{y} I_{F}(y)=$ $\beta \inf _{y \in D} F(y)$. If this infimum is realized at a unique point $y_{*}$ of $D$, the sequence of measures $\mu_{N}$ converges weakly to the Dirac mass $\delta_{y_{*}}$. We thus look for the minima of $F$ on $D$.

$$
\begin{gathered}
\inf _{\left(m^{+}, m^{-}\right) \in D} F\left(m^{+}, m^{-}\right) \\
=\inf _{|m| \leqslant 1}\left(G(M)+b_{G C} m+\left(b_{A T}-b_{G C}\right) \sup _{\left(m^{+}, m^{-}\right): m^{-}+m^{+}=m} m^{+}\right)
\end{gathered}
$$

Given, $\rho^{+}$, consider $m$ such that $M=(m+1) / 2 \geqslant \rho^{+}$, that is $m \geqslant 2 \rho^{+}-1$. Then $\sup _{m^{+}+m^{-}=m} m^{+}=\rho^{+}($corresponding to (10) below) $)$: when $m^{+}=$ $\rho^{+}$, one obtains $m^{-}=m-\rho^{+}$and the pair $\left(m^{+}, m^{-}\right)$is element of $D$ since $m^{-}=m-\rho^{+} \leqslant \rho^{-}$if and only if $m \leqslant \rho^{+}+\rho^{-}=1$ and $m^{-} \geqslant-\rho^{-}$if and only if $m \geqslant \rho^{+}-\rho^{-}=2 \rho^{+}-1$ Similarly, when $m$ is such that $M \leqslant \rho^{+}$, the maximal possible value of $m^{+}$is $m^{+}=2 M-\rho^{+}$(corresponding to (11) below). In summary the infimum is obtained by taking the minimum between

$$
\inf _{M \geqslant \rho^{+}} G(M)+b_{G C} m+\left(b_{A T}-b_{G C}\right) \rho^{+}
$$

and

$$
\inf _{M<\rho^{+}} G(M)+b_{A T} m+\rho^{-}\left(b_{A T}-b_{G C}\right) .
$$

We next use the hypotheses. From Theorem 2$] \kappa<\kappa_{c}\left(B_{A T}\right)$ implies that $G(M)+b_{A T} m$ attains its infimum when $m_{*}=1$, or $M_{*}=1$, and is decreasing on the unit interval; (11) becomes

$$
G\left(\rho^{+}\right)+b_{A T}\left(2 \rho^{+}-1\right)+\left(1-\rho^{+}\right)\left(b_{A T}-b_{G C}\right)=G\left(\rho^{+}\right)+\rho^{+} b_{A T}-\rho^{-} b_{G C} .
$$

Concerning (10), $\kappa>\kappa_{c}\left(B_{G C}\right)$ and, from Theorem 2 the function $G(M)+$ $b_{G C} m$ attains its minimum at $y_{*}\left(B_{G C}, \kappa\right)$, and is increasing above this point. Thus

$$
\inf _{M \geqslant \rho^{+}} G(M)+b_{G C} m=G\left(\rho^{+}\right)+\left(2 \rho^{+}-1\right) b_{G C},
$$

when $\rho^{+} \geqslant y_{*}\left(B_{G C}, \kappa\right)$. Then, both (10) and (11) are minimized for $M=\rho^{+}$, which is the maximal value of $m^{+}$. Thus $m^{+}=\rho^{+}$and $m^{-}=m-m^{+}=$ $2 \rho^{+}-1-\rho^{+}=-\rho^{-}$, as required. 
Conversely, assume that $\rho^{+}<y_{*}\left(B_{G C}, \kappa\right)$. Then (10) becomes

$$
\begin{gathered}
\inf _{M \geqslant \rho^{+}} G(M)+b_{G C} m+\left(b_{A T}-b_{G C}\right) \rho^{+} \\
=G\left(y_{*}\left(B_{G C}, \kappa\right)\right)+b_{G C}\left(2 y_{*}\left(B_{G C}, \kappa\right)-1\right)+\left(b_{A T}-b_{G C}\right) \rho^{+} .
\end{gathered}
$$

When $M<\rho^{+}$, the infimum is still given by

$$
G\left(\rho^{+}\right)+b_{A T} \rho^{+}-\rho^{-} b_{G C}=G\left(\rho^{+}\right)+b_{G C}\left(2 \rho^{+}-1\right)+\rho^{+} b_{A T}-\rho^{+} b_{G C},
$$

and one obtains that the minimum is realized when $M=y_{*}\left(B_{G C}, \kappa\right)$, and therefore $m^{+}=\rho^{+}$and $m^{-}=m-m^{+}=2 M-1-m^{+}=2 y_{*}\left(B_{G C}, \kappa\right)-1-\rho^{+}$, as required.

In the remaining, we give an example of probability measure $V_{N}$ on $\Omega_{N}$ such that $Q_{N}=V_{N} \circ \Psi_{B^{N}}^{-1}$ satisfies a large deviation principle with rate function $I_{D}$. Given a sequence $\left(B^{N}\right)$, consider the family of spins $\sigma^{N}$ given by $\sigma_{i}^{N}=\mathrm{I}\left(b_{i}^{N}=b_{A T}\right)-\mathrm{I}\left(b_{i}^{N}=b_{G C}\right)$. Given a sequence $r_{N}$, consider the restricted Ising measure

$$
V_{N}(\sigma)=\pi_{\beta_{a}, r_{N}}(\sigma)=\frac{\mathrm{I}\left(|\sigma| \leqslant 2 r_{N}\right) \pi_{\beta_{a}}(\sigma)}{\sum_{\sigma \in \Omega_{N}} \mathrm{I}\left(|\sigma| \leqslant 2 r_{N}\right) \pi_{\beta_{a}}(\sigma)} .
$$

Lemma 2 Let $\left(B^{N}\right)_{N \in \mathbf{N}}$ be a sequence of words such that $\rho_{N}^{+} \longrightarrow \rho^{+} \in \mathrm{I}$, and $\sigma^{N} \in \mathcal{C}_{\bar{r}_{N}, N}$, for some sequence $\left(\bar{r}_{N}\right)_{N \in \mathbf{N}}$. Assume that $\bar{r}_{N}+2 \leqslant r_{N}$, and that $r_{N} / N \longrightarrow 0$ as $N \rightarrow \infty$. Then the probability measure $Q_{N}=$ $\pi_{\beta_{a}, r_{N}} \circ \Psi_{B^{N}}^{-1}$ satisfies a large deviation principle with rate function $I_{D}$.

This is an extension of the homopolymer case: $\bar{r}_{N} / N \rightarrow 0$ means that the word associated with the DNA is formed of relatively large droplets of $\mathrm{A}+\mathrm{T}$ bonds alternating with similar droplets of $\mathrm{G}+\mathrm{C}$ bonds.

Proof: When $r_{N} / N \rightarrow 0$, (8) implies that $\log \left(V_{N}\left(\tilde{\sigma}^{N}\right)\right) / N \rightarrow 0$ for any sequence of spins $\left(\tilde{\sigma}^{N}\right)_{N}$ with $\left|\tilde{\sigma}^{N}\right| \leqslant 2 r_{N}$. Now, given an open subset $A$ of $D^{0}$, containing some point $\lambda=\left(\lambda^{+}, \lambda^{-}\right)$, with $\left|\lambda^{+}\right|<\rho^{+}$and $\left|\lambda^{-}\right|<\rho^{-}$, consider the sequence $\lambda_{N}=\left(\left[N \lambda^{+}\right] / N,\left[N \lambda^{-}\right] / N\right)$, which is in $A$ for $N \geqslant$ $N_{0}(A, \lambda)$. Then

$$
\exists \tilde{\sigma}^{N} \in \mathcal{C}_{\tilde{r}_{N}, N} \text { with } \tilde{r}_{N} \leqslant \bar{r}_{N}+1 \text { and } \Psi_{B^{N}}\left(\tilde{\sigma}^{N}\right)=\lambda_{N}, \forall N \geqslant N_{0}(A, \lambda) .
$$

Suppose that (12) is true: Then one obtains

$$
V_{N}\left(\tilde{\sigma}^{N}\right) \leqslant Q_{N}\left(\left\{\lambda_{N}\right\}\right) \leqslant Q_{N}(A) \leqslant 1
$$

and it follows that $\log \left(Q_{N}(A)\right) / N \rightarrow 0$ as $N \rightarrow \infty$. In this case, $A$ is a $I_{D^{-}}$ continuity set. The main property to check is thus (12). Set $\lambda_{N}^{ \pm}=\left[\lambda^{ \pm} N\right] / N$, 
and define $M_{N}^{ \pm}=\left(\lambda_{N}^{ \pm}+\rho_{N}^{ \pm}\right) / 2$, where $\rho_{N}^{-}=1-\rho_{N}^{+}$. Choose an origin in the circular DNA at some site $i_{0}$ with $\sigma_{i_{0}-1}^{N}=-1$ and $\sigma_{i_{0}}^{N}=1$. We can consider the linear string, starting at $i_{0}$, with an $\mathrm{A}+\mathrm{T}$ droplet, and ending with a $\mathrm{G}+\mathrm{C}$ droplet. Let $A_{1}, \cdots, A_{\bar{r}_{N}}$ be the $\mathrm{A}+\mathrm{T}$ droplets, ordered according to their appearance along the string, and define similarly $G_{1}, \cdots, G_{\bar{r}_{N}}$. Set $a_{j}=$ $\left|A_{j}\right|$ and $g_{j}=\left|G_{j}\right|, 1 \leqslant j \leqslant \bar{r}_{N}$. The string is viewed as the juxtaposition of symbols $A_{1} G_{1} \cdots A_{\bar{r}_{N}} G_{\bar{r}_{N}}$. Let $T^{+}$and $T^{-}$be defined by

$$
T^{+}=\min \left\{1 \leqslant j \leqslant \bar{r}_{N} ; \sum_{k=1}^{j} a_{k} \geqslant N M_{N}^{+}\right\},
$$

and

$$
T^{-}=\min \left\{1 \leqslant j \leqslant \bar{r}_{N} ; \sum_{k=1}^{j} g_{k} \geqslant N M_{N}^{-}\right\} .
$$

Notice that both $T^{+}$and $T^{-}$are well defined for $N$ large enough since $\sum_{k=1}^{\bar{r}_{N}} a_{k}=N \rho_{N}^{+}, \sum_{k=1}^{\bar{r}_{N}} g_{k}=N \rho_{N}^{-}, \rho_{N}^{+} \rightarrow \rho^{+}, \rho_{N}^{-} \rightarrow \rho^{-}, M_{N}^{+} \rightarrow\left(\lambda^{+}+\right.$ $\left.\rho^{+}\right) / 2<\rho^{+}$and $M_{N}^{-} \rightarrow\left(\lambda^{-}+\rho^{-}\right) / 2<\rho^{-}$. Let $i_{1}$ be the site situated in $A_{T^{+}}$such that

$$
\left|\left\{i \in A_{1} \cup \cdots \cup A_{T^{+}} ; i \leqslant i_{1}\right\}\right|=N M_{N}^{+},
$$

and define similarly $i_{2}$ for the $\mathrm{G}+\mathrm{C}$ domain. Set $\tilde{\sigma}_{i}^{N}=+1$ when $i \in A_{1} \cup$ $\cdots \cup A_{T^{+}}$and $i \leqslant i_{1}, \tilde{\sigma}_{i}^{N}=-1$ when $i \in A_{T^{+}} \cup \cdots \cup A_{\bar{r}_{N}}$ and $i>i_{1}, \tilde{\sigma}_{i}^{N}=-1$ when $i \in G_{1} \cup \cdots \cup G_{T^{-}}, i \leqslant i_{2}$, and $\tilde{\sigma}_{i}^{N}=+1$ when $i \in G_{T^{-}} \cup \cdots \cup G_{\bar{r}_{N}}$, $i>i_{2}$. Clearly

$$
\begin{aligned}
\sum_{i \in \cup_{1 \leqslant k \leqslant \bar{r}_{N}} A_{k}} \tilde{\sigma}_{i}^{N} & =N M_{N}^{+}-\left(N \rho_{N}^{+}-N M_{N}^{+}\right) \\
& =N\left(2 M_{N}^{+}-\rho_{N}^{+}\right)=N \lambda_{N}^{+},
\end{aligned}
$$

and similarly

$$
\sum_{i \in \cup_{1 \leqslant k \leqslant \bar{r}_{N}} G_{k}} \tilde{\sigma}_{i}^{N}=N \lambda_{N}^{-},
$$

giving $\Psi_{B^{N}}\left(\tilde{\sigma}^{N}\right)=\lambda_{N}$, as required. Next suppose without loss of generality that $T^{+} \leqslant T^{-}$, then $i_{1}<i_{2}$, and, from construction, $\tilde{\sigma}_{i}^{N}=\sigma_{i}^{N}$ when $i \leqslant i_{1}$, $\tilde{\sigma}_{i}^{N}=-\sigma_{i}^{N}$ when $i>i_{2}$ and $\tilde{\sigma}_{i}^{N}=-1$ when $i_{1}<i \leqslant i_{2}$. It follows that $\left|\tilde{\sigma}^{N}\right| \leqslant 2\left(\bar{r}_{N}+1\right)$, as required.

Now, consider a Borel subset $A \subset D^{c}$ in $\mathbf{R}^{2}$. For any sequence of spins $\left(\tilde{\sigma}^{N}\right)$ in $\Omega_{N},-\rho_{N}^{ \pm} \leqslant m_{N}^{ \pm}\left(\tilde{\sigma}^{N}\right) \leqslant \rho_{N}^{ \pm}$, and thus $\Psi_{B^{N}}\left(\tilde{\sigma}^{N}\right) \in A^{c}$ for $N$ large enough, that is there exists $N_{0}(A) \in \mathbf{N}$ such that $Q_{N}(A)=0, \forall N>N_{0}(A)$. 


\section{Statistical approach}

\subsection{A Bayesian model}

In the heteropolymer case, the field $B^{N}=\left(b_{i}\right)$ is indexed by a word of length $N$ on the two letters alphabet $\{A+T, G+C\}$. The DNA can be seen as a kind of geometrical code where a word is glued on the double helix. When the perimeter is fixed by setting $V_{N}=U_{r, N}, \mathcal{C}_{r, N}=\left\{\sigma \in \Omega_{N} ;|\sigma|=2 r\right\}$ is a nonlinear code, as a subset of $\Omega_{N}$, and tools from information theory and bayesian statistics are of great utility for computational issues. We will see that the Hamiltonian of the system models the a posteriori law on the parameter space $\Omega_{N}$ given the observation $B^{N}$. First, consider the a priori probability measure on the parameter space given by

$\nu_{N}(\sigma)=\frac{V_{N}(\sigma) \exp \left(-N \beta G\left(M_{N}(\sigma)\right)\right) \prod_{i=1}^{N}\left(\exp \left(-\sigma_{i} \beta b_{A T}\right)+\exp \left(-\sigma_{i} \beta b_{G C}\right)\right.}{Z_{N}\left(\nu_{N}\right)}$,

where $Z_{N}\left(\nu_{N}\right)$ denotes the related partition function and $V_{N}$ is an arbitrary probability measure on $\Omega_{N}$. Notice that $\nu_{N}$ takes into account the level of superhelicity of the DNA through the superhelical density $\kappa$, and that $\nu_{N}$ corresponds to the Gibbs measure associated with the homopolymer with field $\bar{B}$, given by

$$
\bar{b}=\frac{b_{A T}+b_{G C}}{2}
$$

that is

$$
\nu_{N}(\sigma)=\frac{V_{N}(\sigma) \exp \left(-N \beta F_{\bar{B}}(\sigma)\right)}{Z_{N}\left(\beta, \bar{B}, V_{N}\right)} .
$$

The statistical model is as follows: a code word or a parameter $\sigma^{0}$ is chosen at random with law $\nu_{N}$ on $\operatorname{supp}\left(V_{N}\right)$, and is sended through a noisy channel with output alphabet $\{A+T, G+C\}$ and memoryless channel statistics $P\left(\cdot \mid \sigma^{0}\right)$ given by

$$
P\left(B^{N} \mid \sigma^{0}\right)=\prod_{i=1}^{N} p\left(b_{i} \mid \sigma_{i}^{0}\right)=\prod_{i=1}^{N} \frac{\exp \left(-\sigma_{i}^{0} \beta b_{i}\right)}{\exp \left(-\sigma_{i}^{0} \beta b_{A T}\right)+\exp \left(-\sigma_{i}^{0} \beta b_{G C}\right)} .
$$

The output distribution of $B^{N}$ is

$$
q\left(B^{N}\right)=\sum_{\sigma \in \Omega_{N}} \nu_{N}(\sigma) P\left(B^{N} \mid \sigma\right),
$$

and the a posteriori distribution on the parameter space is the Gibbs distribution

$$
\pi_{\beta, B^{N}, V_{N}}(\sigma)=\frac{V_{N}(\sigma) \exp \left(-N \beta F_{B^{N}}(\sigma)\right)}{Z_{N}\left(\beta, B^{N}, V_{N}\right)} .
$$


Notice that

$$
\mathrm{E}_{q}\left(\rho_{N}^{+}\left(B^{N}\right)\right)=1-\theta+(2 \theta-1)<M_{N}>_{\nu_{N}},
$$

where $\theta=\exp \left(-\beta b_{A T}\right) /\left(\exp \left(-\beta b_{A T}\right)+\exp \left(-\beta b_{G C}\right)\right) \geqslant 1 / 2$. The law of $M_{N}(\sigma)$ under $\nu_{N}$ is subject to threshold phenomenon, as shown in Section 2.1. When $V_{N}=U_{r_{N}, N}$ with $r_{N} / N \rightarrow 0$, Theorem 2 gives information on the limiting support of $\nu_{N}: \kappa_{c}(\bar{B})<\kappa<\bar{\kappa}_{c}(\bar{B})$ implies that the law of $M_{N}$ under $\nu_{N}$ converges toward the point mass $\delta_{y_{*}(\bar{B}, \kappa)}$ with $y_{*}(\bar{B}, \kappa) \in$ $(0,1)$, and the average proportion of $\mathrm{A}+\mathrm{T}$ bonds in $B^{N}$ under $q$ is given by $1-\theta+(2 \theta-1) y_{*}(\bar{B}, \kappa) \in[1-\theta, \theta]$.

Having observed some word $B^{N}$, consider the Bayes estimator for $\sigma^{0}$ under quadratic loss $L\left(\sigma, \sigma^{\prime}\right)=\left\|\sigma-\sigma^{\prime}\right\|^{2}$. Bayesian Theory (see e.g. Robert (1992)) gives that the optimal Bayes estimator under quadratic loss is

$$
\widehat{\sigma}_{i}=\frac{\sum_{\sigma \in \mathcal{C}_{r, N}} \sigma_{i} \nu_{N}(\sigma) P\left(B^{N} \mid \sigma\right)}{\sum_{\sigma \in \mathcal{C}_{r, N}} \nu_{N}(\sigma) P\left(B^{N} \mid \sigma\right)},
$$

and the estimated magnetization $\sum_{i} \widehat{\sigma}_{i} / N$ corresponds to our order parameter $<m_{N}>_{\pi_{\beta, B}{ }^{N} V_{N}}$, showing that Benham's model has an interesting statistical content.

In the next Section, we imbeed Benham's model in a bayesian segmentation model of current use in bioinformatics, and provide new algorithms with priors adapted to supercoiled DNA.

\subsection{Bayesian segmentation for strand separation}

\subsubsection{A two coins example}

Liu and Lawrence(1999) present a Bayesian model for segmentation of biopolymers, and provide algorithms for drawing samples from the various posterior laws of the model, which might be of great interest in the strand separation problem. We start with their two types of coins example, to fix ideas. Suppose you know that in a coin tossing game of length $N$, the first $A$ Bernoulli have a probability of success $\theta_{1}$ and the next $N-A$ tosses have a probability $\theta_{2} \neq \theta_{1}$ of getting a head. Let $y_{o b s}$ be the observed sequence, whith $h_{1}$ (resp. $h_{2}$ ) heads and $t_{1}$ (resp. $t_{2}$ ) tails in the first (resp. second) part of the sequence. The change point $A$ is treated as a missing data, and has some prior law $g(a)$. The likelihood of the observed data is given by

$$
L\left(\theta_{1}, \theta_{2} ; y_{o b s}, A=a\right)=\theta_{1}^{h_{1}}\left(1-\theta_{1}\right)^{t_{1}} \theta_{2}^{h_{2}}\left(1-\theta_{2}\right)^{t_{2}} g(a) .
$$

In their work, the prior $\pi(\theta)$ for $\theta=\left(\theta_{1}, \theta_{2}\right)$ is a product measure associated with two independent Beta random variables $B\left(\theta_{1} ; \alpha_{1}, \beta_{1}\right)$ and $B\left(\theta_{2} ; \alpha_{2}, \beta_{2}\right)$. Let $p\left(\theta_{1}, \theta_{2}, a, y_{o b s}\right)$ be the joint law of all variables, with

$$
p\left(\theta_{1}, \theta_{2}, a, y_{o b s}\right)=L\left(\theta_{1}, \theta_{2} ; y_{o b s}, A=a\right) \pi(\theta) g(a),
$$


and

$$
P\left(A=a, y_{o b s}\right)=\iint p\left(\theta_{1}, \theta_{2}, a, y_{o b s}\right) \mathrm{d} \theta_{1} \mathrm{~d} \theta_{2} .
$$

The following algorithm will converge and give samples from the posterior law of $\left(\theta_{1}, \theta_{2}, A\right)$ :

- Fix $A=a$ and $\theta_{2}$, and draw $\theta_{1}$ from its conditional posterior law $P\left(\theta_{1} \mid \theta_{2}, A=a, y_{o b s}\right)$, to get the new $\theta_{1}$,

- proceed similarly for $\theta_{2}$ to get the new $\theta_{2}$,

- draw $A$ from its conditional posterior law $P\left(A=a \mid \theta, y_{o b s}\right)$ proportional to $\prod_{i=1,2} \theta_{i}^{h_{i}(a)}\left(1-\theta_{i}\right)^{t_{i}(a)} g(a)$, where $h_{i}(a)$ and $t_{i}(a)$ are the number of heads and tails contained in the $i$-th part of the sequence, $i=1,2$.

Coming back to the setting of Section 3.1 choose $V_{N}$ to be $U_{1, N}$, fixing the perimeter to $2 r=2$. Benham's model deals with a circular DNA, and they are $N$ configurations of length $a$, and two change points. Forgetting for a while this slight difference, we see that the Bayesian model of Liu and Lawrence can be adapted for Benham's model: $\sigma$ is the missing data or segmentation parameter $A$, and the prior law $g(a)$ is just the a priori measure $\nu_{N}$ of Section 3.1 Suppose that $\sigma_{i}=+1,1 \leqslant i \leqslant A$ and $\sigma_{i}=-1$, $A<i \leqslant N$. Set $B^{N}=y_{o b s}$ and fix the parameter $\theta$ to

$$
\bar{\theta}_{1}=\frac{\exp \left(-\beta b_{A T}\right)}{\exp \left(-\beta b_{A T}\right)+\exp \left(-\beta b_{G C}\right)}, \bar{\theta}_{2}=\frac{\exp \left(\beta b_{A T}\right)}{\exp \left(\beta b_{A T}\right)+\exp \left(\beta b_{G C}\right)}=1-\bar{\theta}_{1} .
$$

The model of Section 3.1 can be seen as a particular case of the segmentation model, and the a posteriori law of the change point $A$ is the Gibbs measure $\pi_{\beta, B^{N}, V_{N}}$. For the deterministic model with constant $\bar{\theta}$, the last step of the algorithm corresponds to sampling with $\pi_{\beta, B^{N}, V_{N}}$. Fye and Benham(1999) give algorithms for strand separation using transfer matrices from statistical mechanics; the method can be applied, thanks to the quadratic form appearing in the prior $\nu_{N}$ (the transfer matrices have complex entries, a consequence of the gaussian transform). The perimeter is not limited, and is penalized by the exponential weight appearing in the one dimensional Ising Boltzman weight $\exp (-4 r \beta)$ for a perimeter of $2 r$ (notice that this way of penalizing too large perimeters was implemented in a recent work of Ramensky et altri(2000,2001)). Their method is applicable to situations where the free energies for strand separation $b_{A T}$ and $b_{G C}$ fluctuate: in real situations, chemical reactions can alter these free energies. In the randomized case, the Bayesian segmentation model can also provide an interesting alternative for studying the strand separation problem with random free energies $b_{A T}$ and $b_{G C}$. 


\subsubsection{The general case}

We adapt the setting of Liu and Lawrence(1999) and Ramensky et altri(2000,2001), and indicate the main features of the model. The number of domains or segments of the circular DNA can be limited to $r_{\max }$. The missing data is the spin $\sigma$, with prior law $\nu_{N}$, given by $V_{N}$ and the homopolymer of field $\bar{b}=\left(b_{A T}+b_{G C}\right) / 2$, with free energies $b_{A T}$ and $b_{G C}$ given by formula (11). $\sigma$ can be seen as a juxtaposition of droplets of \pm spins arranged around the discrete circle of length $N$.

Suppose they are $2 r$ domains with $r$ positive droplets, that is containing sites $i$ with $\sigma_{i}=+1$, and $r$ negative droplets. The parameter $\theta$ is here defined given $\sigma$. We associate to every positive droplet $\Lambda_{i_{0}}^{+}$with $\sigma_{i_{0}-1}=-1$, $\sigma_{i}=+1, i_{0} \leqslant i \leqslant i_{k}$, and $\sigma_{i_{k}+1}=-1$ a family of $k+1$ i.i.d. Bernoulli $\varepsilon_{i}$, with

$$
P\left(\varepsilon_{i}=A+T\right)=\theta^{i_{0}, i_{k}} \text { and } P\left(\varepsilon_{i}=G+C\right)=1-\theta^{i_{0}, i_{k}},
$$

of random parameter $\theta^{i_{0}, i_{k}}$, which is chosen according to some prior law $f^{+}$. Do the same for negative droplets for a random parameter $\theta^{j_{0}, j_{l}}$ of prior $f^{-}$, with

$$
P\left(\varepsilon_{j}=A+T\right)=1-\theta^{j_{0}, j_{l}} \text { and } P\left(\varepsilon_{j}=G+C\right)=\theta^{j_{0}, j_{l}} .
$$

The requirements might be

$$
\mathrm{E}_{f^{+}}\left(\theta^{i_{0}, i_{k}}\right)=\mathrm{E}_{f^{-}}\left(\theta^{j_{0}, j_{l}}\right)=\bar{\theta}_{1},
$$

where the constants $b_{A T}$ and $b_{G C}$ appearing in (10) and (13) can be taken as average values. Positive droplets force the sample toward $A+T$ outcomes and conversely for negative droplets. The priors $f^{ \pm}$can be chosen as in Liu and Lawrence(1999) as Beta laws with the required expectations.

A second way of randomizing the parameters consists in taking, independently for each segment, two positive random variables $b_{A T}(\omega)$ and $b_{G C}(\omega)$, distributed according to some law, which might be motivated from thermodynamics or biochemistry, with average values given by formula (11). Draw $2 r$ i.i.d. realizations of these two random variables, and set, for each segment,

$$
\theta(\omega)=\frac{\exp \left(-\beta b_{A T}(\omega)\right)}{\exp \left(-\beta b_{A T}(\omega)\right)+\exp \left(-\beta b_{G C}(\omega)\right)}
$$

The probability to get $A+T$ is $\theta(\omega)$ when the droplet is positive and $1-\theta(\omega)$ when the droplet is negative.

Let $\pi(\theta \mid \sigma)$ be the prior law given by the above construction, with joint law $\pi(\theta, \sigma)=\pi(\theta \mid \sigma) \nu_{N}(\sigma)$. Then the law of $B^{N}$ is

$$
\begin{aligned}
P\left(B^{N}\right) & =\sum_{\sigma \in \Omega_{N}} \nu_{N}(\sigma) P\left(B^{N} \mid \sigma\right) \\
& =\sum_{\sigma \in \Omega_{N}} \nu_{N}(\sigma) \int P\left(B^{N} \mid \theta, \sigma\right) \mathrm{d} \pi(\theta \mid \sigma),
\end{aligned}
$$


where $P\left(B^{N} \mid \theta, \sigma\right)$ is the product measure associated with the Bernoulli. The posterior law of interest in the strand separation problem is just $P\left(\sigma \mid B^{N}\right)=$ $P\left(B^{N} \mid \sigma\right) / P\left(B^{N}\right)$. This last law is define on the cube $\Omega_{N}$, of size $2^{N}$. The observables of interest are the number of denatured bonds $M_{N}(\sigma)$ given by $m_{N}(\sigma)=2 M_{N}(\sigma)-1$, and the restricted magnetization $m_{N}^{+}$and $m_{N}^{-}$. Information on the localization of denaturation can be obtained by considering the proportion of broken $\mathrm{A}+\mathrm{T}$ and $\mathrm{G}+\mathrm{C}$ bonds

$$
M_{N}^{ \pm}=\frac{m_{N}^{ \pm}+\rho_{N}^{ \pm}}{2}
$$

(see Section 2.2). Bayesian segmentation algorithms like backward sampling, as given in Liu and Lawrence(1999) or Schmidler et altri(2000), can thus be applied to the strand separation problem to study local denaturation as function of the various parameters, taking into account the random fluctuations of the free energies needed to denature $\mathrm{A}+\mathrm{T}$ and $\mathrm{G}+\mathrm{C}$ bonds.

\section{References}

R. Baxter. Exactly Solved Models in Statistical Mechanics Academic Press, 1982.

C. Benham(1979) Torsional stress and local denaturation in supercoiled DNA Proc. Natl. Acad. Sci. USA, 76(8):3870-3874

C. Benham(1989) Mechanics and Equilibria of Superhelical DNA. In Mathematical Methods for DNA Sequences Ed. M. Waterman CRC Press.

C. Benham(1990) Theoretical analysis of heteropolymeric transitions in superhelical DNA molecules of specified sequences. J. Chem. Phys., 92(10):62946305

C. Benham(1992) Energetics of the Strand Separation Transition in Superhelical DNA J. Mol. Bio., 225:835-847

C. Benham(1996) Theoretical analysis of the helix-coil transition in positively superhelical DNA at high temperature Phys. Rev. E,53(3):2984-2987.

P. Clote and R. Backofen Computational Molecular Biology: An Introduction Wiley, 2000.

A. Dembo and O. Zeitouni Large Deviations Techniques and Applications Jones and Bartlett, 1992.

J. Deuschel and D. Stroock Large Deviations Academic Press. 1989.

R. Ellis Entropy, Large Deviations and Statistical Mechanics Springer 1985.

W. Feller An Introduction to Probability Theory and Its Applications, Vol. I Wiley, 1971.

R. Fye and C. Benham(1999) Exact method for numerically analyzing a model of local denaturation in superhelically stressed DNA Phys. Rev. E, 
59:3408.

B. Lewis Genes V Oxford University Press. 1994.

J.S. Liu and C.E. Lawrence(1999) Bayesian inference on biopolymer models Bioinformatics 15(1):38-52

J.S. Liu, A.F. Neuwald and C.E. Lawrence Markovian structures in biological sequence alignments Preprint

P. Mathieu and P. Picco(1998). Metastability and convergence to equilibrium for the random field Curie Weiss model. J. Stat. Phys. 91(3/4): 679-732.

V.E. Ramensky, V.J. Makeev, M.A. Roytberg and V.G. Tumanyan(2000) DNA segmentation through the Bayesian approach Journal of Computational Biology. 7(1/2): 215-231

V.E. Ramensky, V.J. Makeev, M.A. Roytberg and V.G. Tumanyan(2001) Segmentation of long genomic sequences into domains with homogeneous composition with BASIO software Bioinformatics 17(11):1065-1066.

C. Robert The Bayesian Choice Springer 1992.

R.T. Rockafellar Convex Analysis Princeton University Press. 1972

H. Sun, M. Mezei, R. Fye and C. Benham(1995) Monte-Carlo analysis of conformational transitions in superhelical DNA J. Chem. Phys, 103(19):86538665 .

S.C. Schmidler, J.S. Liu and D.L. Brutlag(2000) Bayesian segmentation of protein secondary structure Journal of Computational Biology. 7(1/2): 233-248 